\title{
Development of a laser-activated mesoporous silica nanocarrier delivery system for applications in molecular and genetic research
}

Lien M. Davidson

Natalia Barkalina

Marc Yeste

Celine Jones

Kevin Coward 


\title{
Development of a laser-activated mesoporous silica nanocarrier delivery system for applications in molecular and genetic research
}

\author{
Lien M. Davidson, Natalia Barkalina, Marc Yeste, Celine Jones, and Kevin Coward* \\ University of Oxford, Nuffield Department of Obstetrics and Gynaecology, Level 3, Women's Centre, John Radcliffe Hospital, Headington, \\ Oxford OX3 9DU, United Kingdom
}

\begin{abstract}
Nanoparticles have revolutionized medical research over the last decade. One notable emerging area of nanomedicine is research developments in the reproductive sciences. Since increasing evidence indicates links between abnormal gene expression and previously unexplained states of infertility, there is a strong impetus to develop tools, such as nanoparticle platforms, to elucidate the pathophysiological mechanisms underlying such states. Mesoporous silica nanoparticles (MSNPs) represent a powerful and safe delivery tool for molecular and genetic investigations. Nevertheless, ongoing progress is restricted by low efficiency and unpredictable control of cargo delivery. Here, we describe for the first time, the development of a laser-activated MSNP system with heat-responsive cargo. Data derived from human embryonic kidney cells (HEK293T) indicate that when driven by a heat-shock promoter, MSNP cargo exhibits a significantly increased expression following infrared laser stimulus to stimulate a heat-shock response, without adverse cytotoxic effects. This delivery platform, with increased efficiency and the ability to impart spatial and temporal control, is highly useful for molecular and genetic investigations. We envision that this straightforward stimuli-responsive system could play a significant role in developing efficient nanodevices for research applications, for example in reproductive medicine. $\odot$ The Authors. Published by SPIE under a Creative Commons Attribution 3.0 Unported License. Distribution or reproduction of this work in whole or in part requires full attribution of the original publication, including its DOI. [DOI: 10.1117/1.JBO.21.11.115002]
\end{abstract}

Keywords: infrared; laser; nanoparticles; mesoporous silica; heat-shock.

Paper 160448RR received Jun. 28, 2016; accepted for publication Oct. 25, 2016; published online Nov. 14, 2016.

\section{Introduction}

Nanoparticles represent an efficient and inexpensive delivery platform for molecular research and medicine, which can directly target physiological pathways in a noninvasive manner to mark, augment, or suppress endogenous functional activity. In particular, mesoporous silica nanoparticles (MSNPs) are universally recognized as a powerful biomedical nanomaterial distinguished for low cytotoxicity across a variety of cell types. ${ }^{1}$ MSNPs are synthetically modified colloidal silica with highly ordered meso-sized pores ( 2 to $50 \mathrm{~nm})^{2}$ and exhibit a suite of favorable characteristics for use as a targeted delivery vector for reproductive biology. These characteristics include high surface-area-to-volume ratio, high loading capacity, both mechanical and thermal stability, and resistance to fluctuations in $\mathrm{pH}^{3}$ Their small size, similar to that of biomolecules, allows for simple integration into physiological cellular processes, such as internalization via endocytosis or selective targeting of endogenous functional pathways. ${ }^{4,5}$ Notably, by manipulating numerous physical and chemical properties such as size, surface charge, polarity, and functional group/ligand attachments, they can be tailored to facilitate cargo loading and target tissue specificity. ${ }^{6,7}$

While nanotechnology has revolutionized medical treatments over the last decade, investigations into genetic interference by exogenous DNA or RNA are often hampered by low delivery

*Address all correspondence to: Kevin Coward, E-mail: kevin.coward@obs-gyn .ox.ac.uk efficiency and a lack of temporal control during later stages of embryogenesis. A pressing goal of current MSNP research is to increase cargo delivery efficiency. ${ }^{8-10}$ Promising progress is being made to develop stimuli-responsive processes for the on-demand release of cargo with spatial, temporal, and dosage control. $^{2,11,12}$ The newest generations of nanoparticles possess sophisticated coatings, such as gate-keeping mechanisms, that are responsive to certain microenvironments, thus permitting the release of cargo only in the presence of specific exogenous stimuli to restrict the off-target release of cargo. ${ }^{13-16}$ Research into the thermo-enhanced release of cargo from mesoporous materials still remains in its infancy, and the limited literature available has relied on complex nonsilica mechanisms to impart heat-responsive properties to the nanocarrier. ${ }^{8,12,15,17,18}$ The development of a thermo-responsive nanocarrier system that utilizes molecular cargo, which can be thermo-enhanced, has not yet been explored. The efficient delivery of heat-responsive molecular cargo would greatly simplify the application of a nontoxic thermo-enhanced MSNP system.

Heat-shock protein (HSP) promoters are increasingly being investigated as a tool for regulating gene expression and thus represent an ideal candidate for imparting thermo-control to MSNP cargo. HSPs are an evolutionally well-conserved protein family whose synthesis is induced in response to different environmental signals, such as ultraviolet light, infrared (IR) light, and chemical exposure. ${ }^{19}$ The inducible nature of HSP promoters allows for temporal control over target gene expression, thus representing a major advantage for in vivo developmental studies. Indeed, previous studies have demonstrated the success of 


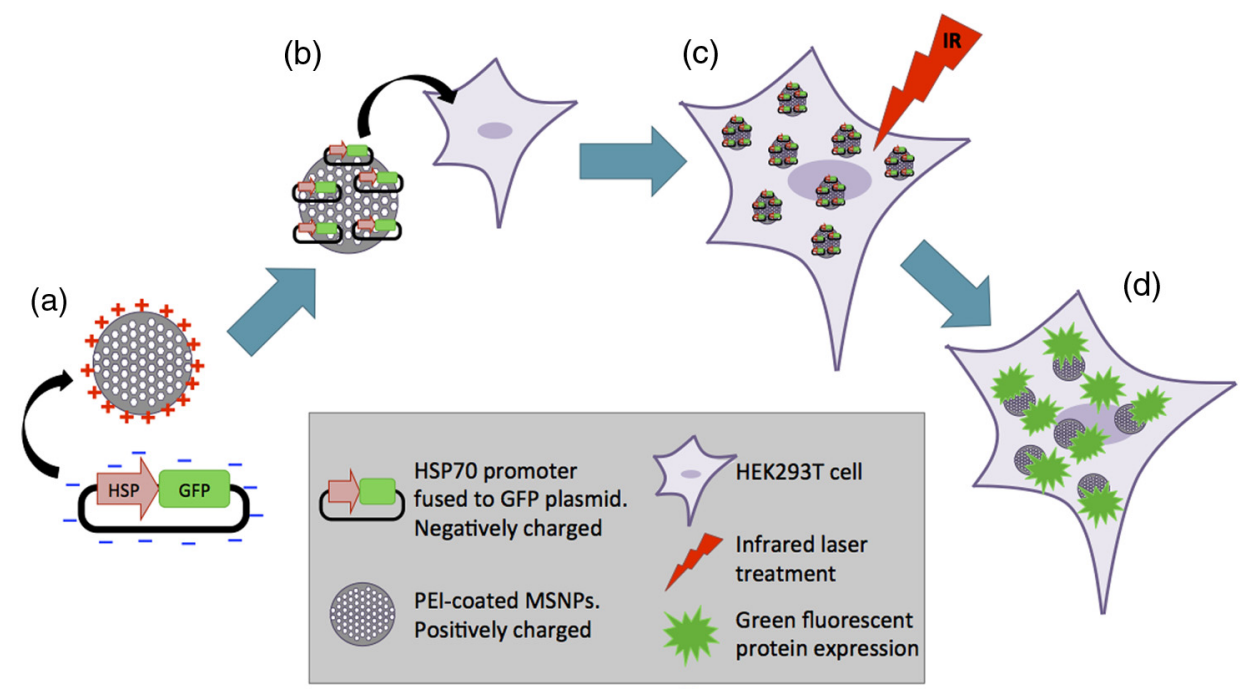

Fig. 1 Schematic showing a stimuli-responsive nanocarrier system activated by IR laser treatment. (a) A heat-shock promoter fused to HSP (HSP:GFP) plasmid with overall negative change electrostatically associated to positively charged polyethyleneimine coated MSNPs. (b) MSNPs loaded with the HSP: GFP plasmid incorporated into HEK293T cells via incubation. (c) HEK293T cells treated with a laser to induce a cellular-heat-shock response. (d) HSP promoter activation to drive the expression of the HSP.

laser irradiation to spatially and temporally induce HSP-mediated expression in transgenic organisms with tissue-specific promoters. $^{20,21}$ However, laser-activated HSP-induction paired with nanoparticle delivery has yet to be explored.

IR lasers are routinely used in assisted reproductive technology (ART) clinics to manipulate sperm, eggs, and embryos for diagnostic and therapeutic purposes. The development of these ART lasers as a specialized research tool is a logical advancement to help elucidate causes of infertility and unknown molecular mechanisms in developmental and reproductive biology. The development of HSP-induced molecular cargo for MSNPs that can be IR laser-activated represents a promising approach with which to increase delivery efficiency while allowing researchers to control temporal and spatial aspects of nanoparticle cargo delivery and activation.

Our study demonstrates the development of an MSNP system whose genetic cargo is under the control of a heat-shock promoter and is activated by IR laser treatment. The laser-activation of specialized nanoparticle cargo using HSP promoters exhibited increased cargo expression from nanocarriers, creating a highly useful tool with which to investigate gene function in developing organisms. Further refinement of this system could provide precise spatial and temporal regulation of cargo delivery in addition to its improved cargo expression efficiency. An effective and finely controlled nanocarrier system with increased efficiency, which can be controlled in both a spatial and temporal manner, could have exciting implications for reproductive science. For example, this refined system could help elucidate causes of unexplained infertility, which are increasingly being associated with abnormal gene expression/genetic polymorphisms but proving difficult to study due to inefficient methods of precisely manipulating gene expression.

\section{Materials and Methods}

This study aimed to generate a mesoporous silica nanocarrier system that is able to deliver molecular cargo that could be activated in target cells by IR laser irradiation. A general schematic depicting the overall experimental design is shown in Fig. 1.

\subsection{Synthesis of Mesoporous Silica Nanoparticles}

Spherical MSNPs with hexagonal pore symmetry $(\sim 130 \mathrm{~nm})$ were synthesized by a surfactant-templated base-catalyzed sol-gel reaction. For a detailed description of MSNP synthesis, characterization, and functionalization, refer to Barkalina et al. ${ }^{22}$ All synthesized MSNPs used in these experiments were functionalized with polyethileneimine (PEI, MW $1.3 \mathrm{kD}$, SigmaAldrich, UK) to impart a positive charge to the surface. For confocal microscopy, MSNPs were functionalized with both PEI and fluorescein isothiocyanate (FITC) to allow fluorescent tracking.

\subsection{Creating the Heat-Shock Promoter-Induced Molecular Cargo}

The thermo-controlled nanoparticle cargo plasmid was created by fusing a heat-shock promoter to the green fluorescent protein (GFP) reporter gene. The 471-bp human HSP70-1 promoter was amplified from the pDRIVE01-hHSP70 plasmid (InvivoGen, San Diego, California) via polymerase chain reaction (PCR) using the High Fidelity PCR master kit (Roche, Germany) and primers designed in-house (forward: 5'-TATACCGGTATGGTGAGCAAGGGCGAG-3' and reverse: 5'-TATGGTACCCTTGTACAGCTCGTCCAT-3'), with a Gstorm Thermal Cycler (Model GS4, GSI, UK). The amplified HSP70-1 promoter was cloned into a linear pcDNA3.1/CTGFP-TOPO plasmid using a GFP fusion TOPO TA expression kit (Life Technologies, UK) according to the manufacturer's protocol. Extraction and purification of plasmid DNA were performed using the QIAprep Spin Miniprep Kit (Qiagen, UK), and DNA yield was increased using the Qiagen Plasmid Maxi Kit (Qiagen, UK). Restriction analysis confirmed appropriate insertion of the target DNA into the plasmid. Cloned plasmids were 
sequenced by Source BioScience (Oxford, UK), and the resultant sequences compared to the HSP70 sequence (pDRIVE01hHSP70 sequence; Invivogen, UK) and GFP sequence (pcDNA3.1/CT-GFP-TOPO sequence; Life Technologies, UK) using ClustalW Multiple Sequence Alignment (BioEdit Sequence Alignment Editor Software version 7.2.5).

\subsection{HEK293T Cell Culture and Maintenance}

Human embryonic kidney (HEK293T) cells were obtained from the American Type Culture Collection (ATCC ${ }^{\circledR} \mathrm{CRL}-3216$ ) and maintained at $37^{\circ} \mathrm{C}$ with $5 \% \mathrm{CO}_{2}$ in a humidified chamber in Dulbecco's Modified Eagle Medium (Sigma-Aldrich, UK) supplemented with $10 \%$ fetal bovine serum, $1 \%$ v/v $5000 \mathrm{U}$ penicillin, $5 \mathrm{mg} / \mathrm{ml}$ streptomycin, and $2 \mathrm{mM} \mathrm{L}$-glutamine and sodium pyruvate. For all experiments, cells were seeded at $30 \%$ confluency on detachable 8-well tissue culture chambers on a polyolefin slide (Sarstedt, Germany).

\subsection{Verification of Heat-Shock Protein: Green Fluorescent Protein Expression}

The cloned HSP:GFP plasmid cargo expression was verified by transfecting $1 \mu \mathrm{g}$ of HSP:GFP plasmid, or $1 \mu \mathrm{l} \mathrm{pcDNA3.1/}$ CT-GFP-TOPO plasmid (without the HSP promoter), into HEK293T cells for $24 \mathrm{~h}$ using jetPEI DNA transfection agent (Polyplus Transfection, France) according to the manufacturer's instruction. To assess cytotoxicity via damage to the plasma membrane, cells were then stained with $10 \mu \mathrm{M}$ propidium iodide (PI; Life Technologies Invitrogen, UK), fixed with $4 \%$ paraformaldehyde (Sigma-Aldrich, UK), and mounted using 4'-6-diamidino-2-phenylinode (DAPI)-containing medium (Vectashield H-1200; DAPI concentration: $1.5 \mu \mathrm{g} / \mathrm{ml}$; Vector Laboratories, UK). HSP:GFP expression and cytotoxicity were analyzed using fluorescence microscopy and data derived from three independent replications of 500 cells.

\subsection{Loading Plasmid Cargo onto Mesoporous Silica Nanoparticles}

Loading of HSP:GFP plasmid onto PEI-coated MSNPs was achieved via electrostatic interaction between the negatively charged nucleic acid and the positively charged cationic surface of PEI-coated MSNPs, as previously described by Barkalina et al. ${ }^{22}$ Briefly, PEI-coated MSNPs were washed by centrifugation and redispersed in nuclease-free water (Ambion, UK). The cloned HSP:GFP plasmid was added to the resulting MSNP at a ratio of 10:1 $\mu \mathrm{g}$ MSNP: $\mu \mathrm{g}$ DNA by mass, and the mixture was incubated for $24 \mathrm{~h}$ at $4^{\circ} \mathrm{C}$ with gentle rotation. MSNPs were recovered through centrifugation and redispersed in $1.0 \mathrm{ml}$ of nuclease-free water. Loading was confirmed by the calculation of free DNA concentration in solution before and after the reaction, based on the measurement of absorbance at $260 \mathrm{~nm}$ by spectrophotometry (BiophotometerPlus, Eppendorf, UK).

\subsection{Mesoporous Silica Nanoparticle Internalization and Cytotoxicity in HEK293T Cells}

To investigate the internalization of MSNPs into HEK cells, fluorescent FITC and PEI-coated MSNPs (FITC-MSNPs) were incubated with HEK293T cells for $24 \mathrm{~h}$ and visualized using confocal microscopy. The signal intensity variance in cells incubated with different FITC-MSNP concentrations (4.0- to 12.0- $\mu \mathrm{g}$ MSNPs $/ 10^{6}$ cells) was assessed using fluorescence microscopy and ImageJ $1.49 \mathrm{v}$ software (National Institute of Health). Next, the cytotoxicity of HSP:GFP-loaded PEI-coated MSNP internalization upon HEK293T cells was determined by incubating cells at $37^{\circ} \mathrm{C}$ with increasing concentrations (4.0- to 12.0- $\mu \mathrm{g}$ MSNPs $/ 10^{6}$ cells), for 24 to $72 \mathrm{~h}$. The MSNP concentration range was derived from previous cytotoxicity studies in our group and publications using similar concentrations in human mammalian cells, which have shown insignificant toxicity. ${ }^{6,23}$ Cells were stained with PI, then fixed and mounted with DAPI for fluorescence imaging, as described earlier, to assess cytotoxicity. Data were derived from three independent replications of 500 cells at each concentration of MSNPs at each time point.

\subsection{Laser Cytotoxicity on HEK293T Cells}

The next stage was to evaluate the potential cytotoxic effects of IR laser treatment upon HEK293T cells, using a Saturn 5 Active laser (1480 nm, $400 \mathrm{~mW}$, Research Instruments, UK), which ordinarily uses a single continuous pulse to deliver a fixed amount of energy. Laser treatment was controlled using RIviewer software (Research Instruments, UK), and within each laser treatment group, all cells assessed were individually laser treated. The laser pulse was delivered to the average center of the cell, which was determined by locating the cross sections of middle of the longest cell length in both the $X$ - and $Y$-axes. HEK293T cells treated with single laser pulses of 10, 20, and $30 \mathrm{~mJ}$ were assessed for cell lysis and morphological changes. A pulse of $30 \mathrm{~mJ}$ was found to lyze the cells and therefore not used in subsequent investigations. Pulses of 10 and $20 \mathrm{~mJ}$ were assessed for cytotoxicity using PI.

Nonlaser-treated cells were used as a control. A pulse of $20 \mathrm{~mJ}$ was selected for further investigation since the heat exclusion zone generated did not lyze the cells and because this energy has been used for other laser-based transfection methods in mammalian cells. ${ }^{24}$ Cells were monitored for 24,48 , and $56 \mathrm{~h}$, stained with PI and DAPI, and fixed and mounted for fluorescence imaging, as described earlier. Data were derived from three independent replications of 100 cells per treatment group. Since neither 10 nor $20 \mathrm{~mJ}$ exhibited cytotoxicity, $20 \mathrm{~mJ}$ was used in subsequent experiments.

\subsection{Laser Activation of Heat-Shock Protein: Green Fluorescent Protein-Loaded Mesoporous Silica Nanoparticles}

Finally, after having optimized the MSNP plasmid loading conditions and confirming that both experimental MSNP concentrations and laser treatments were not cytotoxic to HEK293T cells, the effect of IR laser irradiation upon the induction of transgene expression was assessed. HEK293T cells containing PEI-coated MSNPs loaded with the HSP:GFP plasmid were laser treated with $20 \mathrm{~mJ}$ to assess plasmid expression. Prior to laser treatment, cells were incubated with plasmid-loaded MSNPs for $24 \mathrm{~h}$ to allow MSNP entry and dispersion. Cells were then treated with $20-\mathrm{mJ}$ IR laser irradiation, as described earlier, and cultured for a further $32 \mathrm{~h}$. An additional group of cells incubated with HSP:GFP-loaded MSNPs were not laser treated to act as controls. Cells were stained with PI and DAPI, then fixed and mounted for fluorescence imaging, as described earlier, to analyze HSP:GFP expression. Data were derived from three independent replications of 100 cells per treatment group. 


\subsection{Fluorescence Microscopy}

Fluorescence microscopy was performed using filters for the following wavelengths: 330 to $380 \mathrm{~nm}$ (blue for DAPI-nucleus), 465 to 495 (green for FITC-MSNP and GFP), and 540 to 580 (red for PI-plasma membrane) using a Nikon Eclipse 80i microscope with a Nikon DS-Ril camera and NIS Elements v3.00 software (Nikon, UK), or using a Leica DMIRE2 microscope and camera with HCImage HCI-3268u software (Hamamatsu, Bridgewater, New Jersey). Confocal fluorescent microscopy was performed under oil immersion at a magnification of $100 \times$ using a Becker and Hickl FLIM system mounted on an inverted IX81 Olympus FV1000 FluoView confocal microscope at the following wavelengths: $405 \mathrm{~nm}$ (blue for DAPI-nucleus), $488 \mathrm{~nm}$ (green for FITC-MSNP and GFP), and $559 \mathrm{~nm}$ (red for PI-plasma membrane).

\subsection{Statistical Analysis}

Data were managed using SPSS for Windows (SPSS Inc., Version 21.0, Chicago Illinois) and expressed as mean \pm standard error of the mean (SEM). Data were analyzed by analysis of variance (ANOVA) or repeated measures ANOVA, followed by the Bonferroni posthoc test for pair-wise comparisons. When data were nonnormal, Friedman's test was performed as a nonparametric alternative to repeated measures ANOVA with a Wilcoxon matched-pairs test to evaluate differences between time points, and Kruskal-Wallis test was performed as a nonparametric alternative with Mann-Whitney matched-pairs test to evaluate differences between treatments within each time point. Differences were considered significant at $P \leq 0.05$. Representative graphs were constructed in GraphPad Prism v5.04 (GraphPad Software, La Jolla, California).

\section{Results}

\subsection{Creation of Thermo-Controlled Nanoparticle Cargo}

A thermo-controlled plasmid was successfully created to act as a nanoparticle cargo by fusing the human HSP70 promoter to the GFP reporter gene (HSP:GFP) (Fig. 1). Successful HSP:GFP cloning was confirmed via restriction digestion analysis, and Sanger sequencing showed $100 \%$ alignment between the cloned HSP:GFP (both forward and reverse sequences) and the sequences provided by the respective commercial suppliers. Next, the functionality of the engineered plasmid was determined by transfection into HEK293T cells at $37^{\circ} \mathrm{C}$. HEK293T cells were transfected with the engineered HSP:GFP plasmid, the linear GFP plasmid without the HSP promoter, and without any exogenous DNA treatment for controls. After $24 \mathrm{~h}$ of transfection, GFP expression was only observed in cells that had been transfected with the HSP:GFP plasmid $(P<0.05)$ compared to both linear GFP plasmid and control transfections (Fig. 2).

\subsection{Confirmation of Mesoporous Silica Nanoparticle Internalization into HEK293T Cells}

Successful internalization of MSNPs into HEK293T cells was assessed using fluorescent FITC and PEI-coated MSNPs (FITC-MSNPs) with fluorescent and confocal fluorescent imaging. After 24-h incubation with HEK293T cells, FITCMSNPs revealed a wide variance in fluorescent intensity at all concentrations, with a large number of outlier values evident

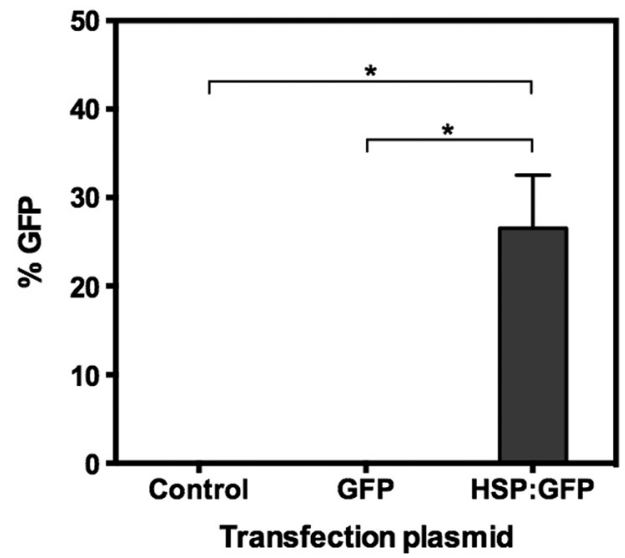

Fig. 2 Mean proportion of transfected cells expressing HSP driven by a heat-shock promoter (HSP:GFP) ( $\% \pm$ SEM). HEK293T cells transfected without DNA for control, $1 \mu \mathrm{g}$ linear GFP plasmid, or $1 \mu \mathrm{g} \mathrm{HSP}$ : GFP cloned plasmid for $24 \mathrm{~h}$. ${ }^{*}$ denotes $P<0.05$. Data derived from three independent replications of 200 cells each.

in most conditions [Fig. 3(a)]. A trend for increased FITC intensity was evident with increasing nanoparticle concentration. The large variance in intensity measurements indicated that not all cells internalized the same amount of MSNPs and that different-sized nanoparticle agglomerates were being formed. Confocal imaging confirmed no fluorescent FITC-MSNP signals in control cells [Fig. 3(b)] and fluorescence in different $z$-planes within the cells incubated with FITC-MSNPs [Fig. 3(c)], confirming that MSNPs had been successfully internalized into cells. Internalization was further demonstrated indirectly in subsequent experiments showing successful expression of the MSNP cargo.

\subsection{Plasmid Cargo Loaded onto Mesoporous Silica Nanoparticles and Delivered into HEK293T Cells Led to Heat-Shock Protein: Green Fluorescent Protein Expression Without Cytotoxicity}

HSP:GFP plasmid cargo was electrostatically loaded into positively charged PEI-coated MSNPs using a previously optimized incubation ratio and time of 10- $\mu \mathrm{g}$ MSNP: $1-\mu \mathrm{g}$ DNA for $24 \mathrm{~h}^{22}$ To assess whether MSNPs were cytotoxic, HEK293T cells were incubated with HSP:GFP-loaded MSNPs at increasing concentrations for up to $72 \mathrm{~h}$ and plasma membrane integrity was examined by PI staining. None of the MSNP concentrations were cytotoxic to HEK293T cells at any time points, with no significant difference in PI staining compared to controls [Fig. 4(a)]. Since HEK293T cells were incubated at $37^{\circ} \mathrm{C}$, straightforward incubation with HEK293T cells with loaded MSNPs resulted in successful plasmid cargo delivery and mild cargo expression [Fig. 4(b)]. Compared to controls, low but significant $(P<0.05)$ HSP:GFP cargo expression was observed in HEK293T cells incubated for $48 \mathrm{~h}$ with 10.0 and $12.0 \mu \mathrm{g} \mathrm{MSNP} / 10^{6}$ cells, and when incubated for $72 \mathrm{~h}$ with 8.0, 10.0, and $12.0 \mu \mathrm{g} \mathrm{MSNP} / 10^{6}$ cells. Cells incubated with $12.0 \mu \mathrm{g} \mathrm{MSNP} / 10^{6}$ cells showed a significantly higher level of GFP expression $(P<0.05)$ compared to controls at 48-, 56-, and 72-h incubation with an increasing trend with time. The highest level of HSP:GFP expression from MSNP incubation alone was $7.29 \%$ after 72-h incubation with $12.0 \mu \mathrm{g} \mathrm{MSNP/}$ $10^{6}$ cells. 


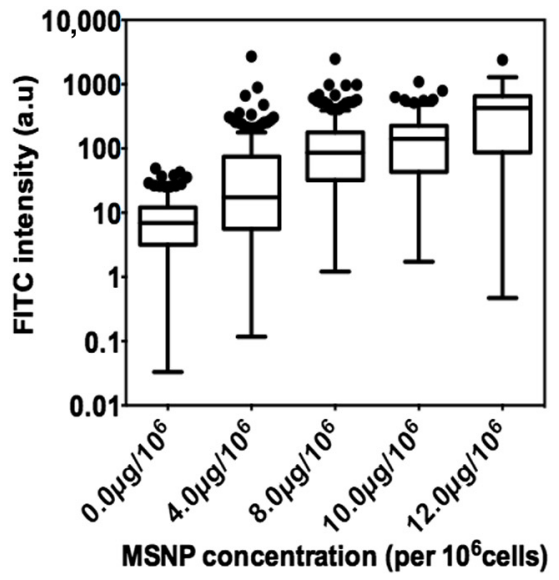

(a)

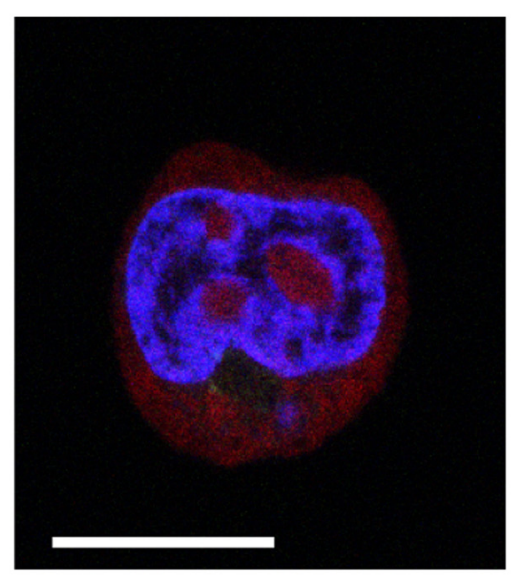

(b)

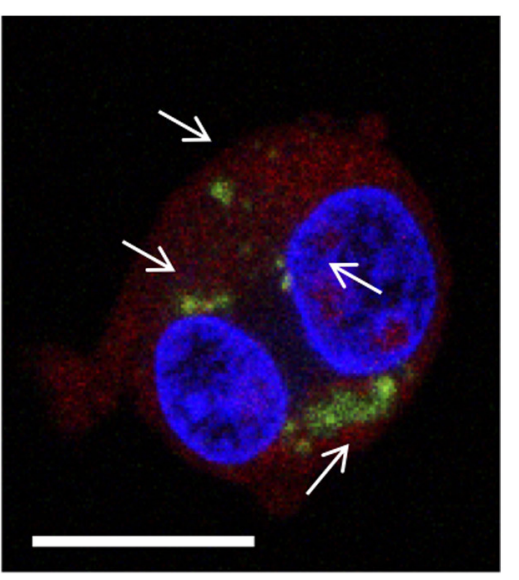

(c)

Fig. 3 MSNP internalization into HEK293T cells. (a) Variance of FITC-MSNP fluorescence intensity (arbitrary units) in HEK293T cells after incubation for $24 \mathrm{~h}$ with increasing concentrations of nanoparticles. Data are depicted on a $\log _{10}$ scale. (b and c) Representative confocal images of HEK293T cells incubated (b) without and (c) with FITC-MSNPs (4.0 $\mu \mathrm{g} \mathrm{MSNP} / 10^{6}$ cells). Images represent composite overlays of stains for nuclei (DAPI: blue), MSNP fluorescence (FITC: green), and plasma membrane integrity (PI: red). White arrows indicate FITC fluorescence. Scale bar $=20 \mu \mathrm{m}$.

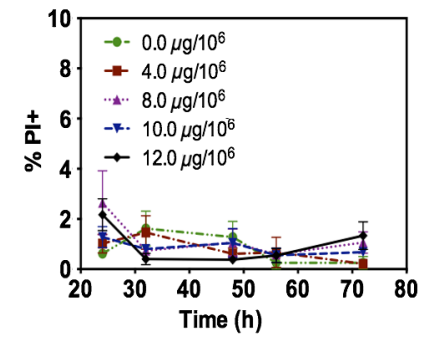

(a)

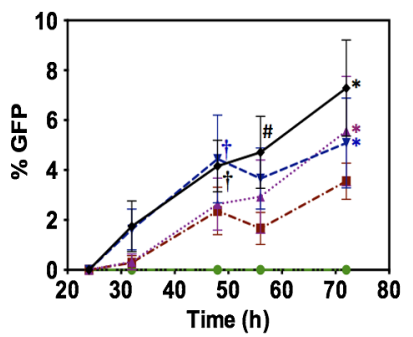

(b)
Fig. 4 HSP:GFP-loaded MSNP cytotoxicity and delivery into HEK293T cells at different concentrations for 24 to $72 \mathrm{~h}$. (a) Mean proportion of cells showing no significant cytotoxicity when assessed by positive PI staining $(\% \pm S E M)$. Data derived from three independent replications of 500 cells. (b) Mean proportion of cells showing HSP:GFP expression from MSNP delivery ( $\% \pm$ SEM). $\dagger$, \#, and * denote significantly different levels of GFP expression between nanoparticles and respective controls $(P<0.05)$ at 48,56 , and $72 \mathrm{~h}$, respectively. Data derived from three independent replications of 500 cells each.

\subsection{Infrared Laser Application Led to the Increased Expression of Plasmid Cargo Delivered into HEK293T cells by Mesoporous Silica Nanoparticles}

IR laser treatment represents an external stimulus, which can induce HSP promoter expression. The potential cytotoxicity of IR laser treatment was analyzed by subjecting HEK293T cells to a 10- or 20-mJ laser pulse to the center of cells. PI staining showed that neither 10- nor 20-mJ IR laser treatment exhibited membrane damage, with membrane integrity competent as early as $24 \mathrm{~h}$ after treatment [Fig. 5(a)]. Application of 20-mJ laser pulse treatment was, therefore, explored as a stimulus to increase HSP:GFP induction in subsequent experiments.

HEK293T cells were incubated with HSP:GFP-loaded MSNPs at a concentration of $12.0 \mu \mathrm{g}$ MSNP $/ 10^{6}$ cells to investigate whether IR laser treatment would induce an elevated HSP response within cells and thus increase the amount of HSP: GFP cargo expressed. After $24 \mathrm{~h}$ of incubation with loaded MSNPs to allow internalization, cells were either treated with a 20-mJ IR laser pulse or left untreated and then left to incubate for a further $48 \mathrm{~h}$. HEK293T cells with unloaded MSNPs served as a control. HEK293T cells incubated with loaded MSNPs with and without laser irradiation both exhibited HSP:GFP expression compared to controls $(P<0.05)$. Notably, while the highest level of HSP:GFP expression from loaded MSNP incubation alone was $7.29 \%$, the level of HSP:GFP expression from lasertreated cells incubated with loaded MSNPs was twofold higher $(14.50 \% ; P<0.05)$ [Figs. 5(b) and 5(c)]. These results indicate that IR laser treatment elicited an elevated heat-shock response and thus increased the expression of nanoparticle cargo and efficiency of MSNP delivery.

\section{Discussion}

In this paper, we have developed an IR laser-activated mesoporous silica nanodelivery platform and carried out feasibility studies in mammalian cells. However, our ultimate goal is to apply this platform to reproductive biomedicine for targeted gene transfer that could assist in the elucidation of mechanisms underlying unexplained infertility and ultimately provide options for diagnosis and therapy. Recent advances in the use of nanocarriers for gene transfer into reproductive tissues, gametes, and embryos are rapidly creating a powerful tool with which to manipulate and study pathophysiological mechanisms in the reproductive sciences. ${ }^{25-27}$ For example, our group has recently demonstrated the ability of MSNPs to bind with mammalian sperm without negative effects upon key semen parameters. ${ }^{22}$ In addition, customization of the aforementioned nanoparticles with a cell-penetrating peptide coating has been shown to increase the rates of association with sperm. ${ }^{28}$ Such developments in the utilization of nanodevices in reproductive medicine are emerging as powerful research tools as well as platforms for potential simultaneous diagnostic and therapeutic ("theranostic") applications. However, to permit more sophisticated 


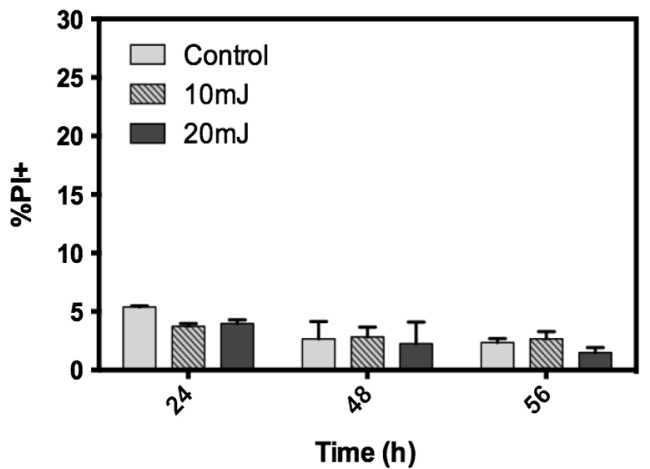

(a)
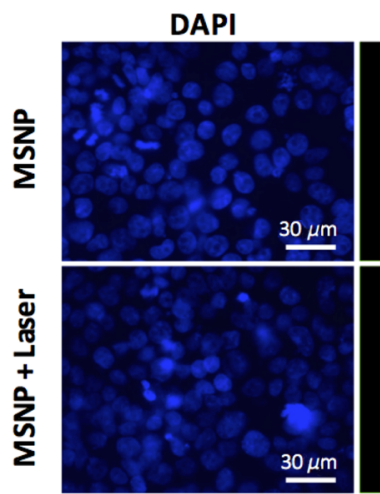

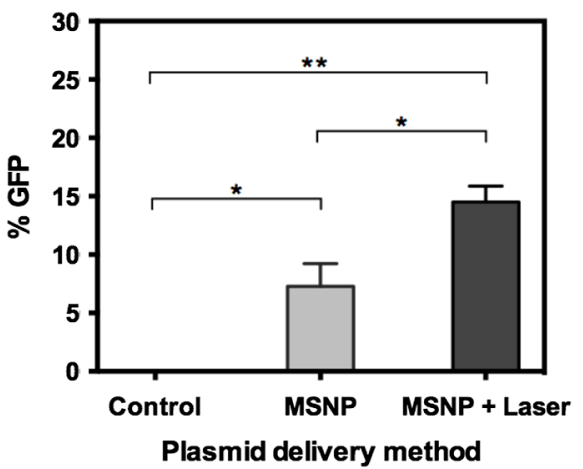

(b)
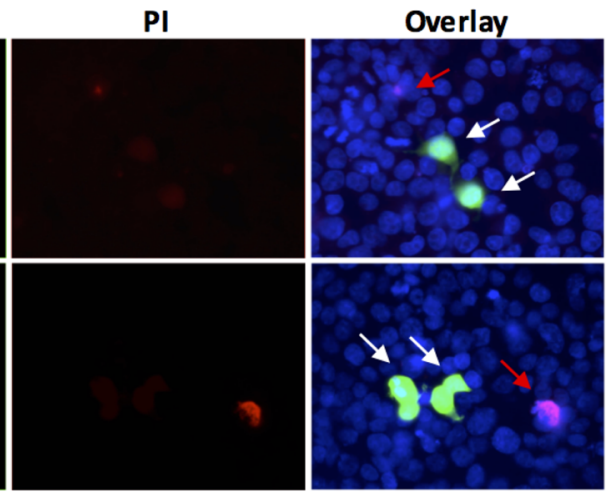

(c)

Fig. 5 Effects of IR laser treatment upon HEK293T cytotoxicity and HSP:GFP MSNP cargo expression. (a) Mean proportion of cells showing no significant cytotoxicity when assessed by positive PI staining (\% \pm SEM) following 10 - or $20-\mathrm{mJ}$ IR laser treatment and subsequent incubation for 24,48 , or $56 \mathrm{~h}$. Data derived from three independent replications of 100 cells each. (b) Mean proportion of cells expressing GFP ( $\% \pm$ SEM) following HSP:GFP-loaded MSNP incubation $\left(12 \mu \mathrm{g} / 10^{6}\right.$ cells) for $72 \mathrm{~h}$ with or without laser stimulus $(20 \mathrm{~mJ})$. ${ }^{*}$ denotes $P<0.05$ and ${ }^{* *}$ denotes $P<0.01$. Data derived from three independent replications of 100 cells each. (c) Representative fluorescence images of laser or nonlaser-treated HEK293T cells incubated with $12 \mu \mathrm{g} / 10^{6} \mathrm{HSP}$ :GFP-loaded MSNPs for $72 \mathrm{~h}$. Images are composite overlays of stains for nuclei (DAPI: blue), plasmid expression (HSP:GFP: green), and compromised plasma membrane (PI: red). White arrows represent cells expressing HSP:GFP. Red arrows represent cells with positive PI staining. Scale bars $=30 \mu \mathrm{m}$. 40× magnification.

research, these delivery systems now require further refinement. Current MSNP systems need to be more efficient in their association/internalization rates, and the ability to control cargo expression/release in a spatial and/or temporal manner would be particularly useful given the highly orchestrated series of events that occur when an egg is fertilized or when an embryo begins to develop.

The risk of systemic and/or local toxicity of engineered nanomaterials is a concern in many cells and tissues, but particularly for gametes in reproductive medicine, where the essential function of these highly specialized cells and sensitive biological systems involved is to safely transmit genetic information to offspring. Since MSNPs are distinguished for their low toxicity even in gametes, they represent a strong candidate for prospective clinical and research applications. ${ }^{3,6,22}$ Nonetheless, an ongoing challenge for MSNP delivery lies in the efficiency of cargo release. A prominent limitation of current stimulienhanced MSNP systems is that they require complicated synthesis or assembly and nonsilica components that do not possess the same levels of biocompatibility. Recent advancements in the temperature-controlled release of molecules from mesoporous materials have been achieved using thermosensitive polymer derivatives. Paraffin, ${ }^{29}$ lipid bilayers, ${ }^{30}$ complex acrylamide polymers, ${ }^{15}$ and acrylic acid copolymers ${ }^{31}$ have been investigated as caps to mesoporous silica shells, which are specifically designed to melt at certain temperatures. Moreover, double stranded DNA sequences, ${ }^{32}$ reversible single stranded DNA, ${ }^{33}$ and coiled peptide motifs ${ }^{34}$ have all been used as valves for MSNPs, which denature at an increased temperature to release encapsulated cargo. Alternating magnetic fields have also been investigated to increase local temperature, leading to pulsatile drug release from nonsilica capped MSNPs. ${ }^{33,35}$ However, these methods all require complex synthesis to load the drug effectively and functionalize valves onto the pores.

The development of a stimuli-responsive nanoparticle system with increased efficiency, which permits the spatial and temporal control of gene expression, would allow for more sensitive and in-depth analysis. MSNPs, a well-established nanocarrier with favorable biocompatibility and loading capacity profiles, were selected as the nanocarriers for development in this study. Clinical-assisted reproduction IR laser systems, such as the one used in this study, have been developed over decades of research and refinement to minimize risk to delicate reproductive tissues, rendering them an ideal candidate for research on 
biological tissue. These laser systems are routinely used on clinical reproductive cells and have been investigated as a research tool for many reproductive models, such as mice and zebrafish embryos. To incorporate a stimuli-responsive aspect into the delivery system, we first developed a thermocontrollable molecular construct with a heat-responsive characteristic via the incorporation of an HSP promoter. Second, we illustrated that this construct could be successfully loaded and delivered as MSNP cargo. Finally, we demonstrated that IR laser treatment could induce a heat-shock response in HEK293T cells containing loaded MSNPs, thereby increasing the expression efficiency of the delivered cargo.

The MSNPs used in these investigations were PEI-coated, a simple functionalization known to be effective for electrostatically loading DNA as nanoparticle cargo, preventing nanoparticle agglomeration, and improving interaction with cells. ${ }^{6,36}$ Data showed that the heat-responsive plasmid was successfully linked via electrostatic association to PEI-coated MSNPs after $24 \mathrm{~h}$ of incubation, thus representing a highly suitable cargo for nanoparticle delivery. These results support previous studies reporting the effectiveness of coating MSNPs with PEI to bind nucleic acids. ${ }^{5,36,37}$ Our current investigations further demonstrated successful internalization of loaded MSNPs by HEK293T cells, demonstrating cargo expression without additional intervention. This also concurs with a series of earlier studies, ${ }^{2,13,38}$ which independently reported the association of MSNPs to HEK293T cells.

Evidence in the literature has shown the effectiveness of heatshock promoters, particularly HSP70, at inducing gene expression in response to external stressors in a variety of different models..$^{20,39-41}$ To the best of our knowledge, there are presently no reports describing the use of molecular cargo driven by an HSP response to improve cargo expression by MSNP delivery in a thermodynamic manner. The use of DNA cargo itself as the responsive element is highly beneficial due to its naturally high cellular uptake and biocompatibility compared to the other responsive elements commonly associated with nanoparticles, such as gold spheres or chemical valves. ${ }^{33,42}$

Previous reports have illustrated spatially controlled laser-activated HSP promoter-induced genes in transgenic models. ${ }^{43-45}$ However, to the extent of our knowledge, the use of an HSP construct as cargo for MSNPs that can be thermally triggered by IR laser irradiation to increase expression has not yet been reported. We thus investigated, for the first time, the use of IR laser irradiation of cells containing HSP cargo-loaded MSNPs as a method of inducing a cellular heat-shock response to specifically increase expression of the cargo. We hypothesized that an external laser stimulus would promote a cellular heat-shock response causing increased transcription of genes driven by a heat-shock promoter and thus the expression of our cargo. GFP expression from HEK293T cells incubated with HSP:GFP-loaded MSNPs was twofold higher $(P<0.05)$ with the application of laser stimulus, compared to no laser treatment, indicating that the plasmid cargo being carried by the MSNPs were successfully IR laser activated, which has never before been shown. An explanation for this increased cargo expression is that the IR laser stimulus is successfully inducing a cellular heat-shock response. Heat-inducible transcription of HSP70 is mediated by heat-shock transcription factors (HSFs), which are synthesized constitutively but remain mostly dormant under nonstressed conditions. In response to heatshock, HSFs trimerize and bind with high affinity to heat- shock promoter elements found upstream of stress protein genes, resulting in the transcription of HSPs. ${ }^{46}$ The increased level of gene expression observed in our experiment is, therefore, likely due to more HSFs being activated by the laser stimulus. This represents a highly promising technique for increasing the gene expression of other molecular constructs in a thermo and spatially controlled manner for molecular investigations and gene therapy. In the future, laser irradiation could be applied to manipulate gene function in vivo, where increased expression efficiency and spatial control are required. In addition to increased expression, finely tuned spatial control imparted by laser stimulus could help solve persistent problems of gene expression in nontarget cells, which often occurs in existing techniques such as mosaic analysis. ${ }^{20}$

To date, only a limited number of studies have reported the use of IR or near-IR (NIR) laser treatment with mesoporous silica; however, they require the use of gold cores and the associated plasmonic resonance in response to the laser photons. For example, Volodkin et al. ${ }^{47}$ demonstrated that gold cores inside MSNP pores could be heated with NIR light to transfer heat to liposome bilayer cargo to release encapsulated dye molecules, while Mura et al. ${ }^{48}$ reported that an increase in temperature of NIR-treated gold nanoparticle cores caused a phase transition in polymers capping mesoporous silica shells, allowing leakage of a preloaded drug. Chen et al. ${ }^{49}$ further illustrated that mesoporous silica shells encapsulating gold nanorod cores provided mechanical support against thermal deformation following laser irradiation, compared to uncoated nanorods. While these reports used NIR treatment with mesoporous silica components, it was the gold particles that were responsible for thermoresponsiveness through plasmon resonance and two-photonactivated therapy of photosensitizers. Such investigations are useful for strategies to eradicate or harm tissues, e.g., in cancer applications,${ }^{50}$ but are not suitable for applications in highly specialized reproductive cells and tissues, which need to safely transmit genetic information to future generations. ${ }^{51}$ It is known that the toxicity of gold nanomaterials varies widely and in a dose-dependent manner. ${ }^{52}$ Additionally, gold nanostructures are known to irreversibly deform under even very lowenergy stimulus, resulting in a spectral shift of peak surface plasmon resonance absorption, thereby reducing the efficiency of any photon or laser-induced methods, and have also been shown to be cytotoxic in mammalian cells following laserinduced modifications. ${ }^{49,53}$ In contrast, our present study investigated the use of MSNPs alone with IR laser treatment to increase the expression of MSNP cargo naturally via normal cellular machinery, illustrating that a heat-stimulus response can be achieved without the use of two-photon-activation using gold cores, which is highly desirable for investigations in the reproductive sciences.

While a study by Andersson et al. ${ }^{54}$ explored the potential use of a laser-induced heat-shock response to activate HSP: GFP plasmid expression, these authors did not use a nanoparticle platform to deliver these constructs. Instead, they used transfection to deliver the construct into HeLa cells and then injected the cells along with gold nanorods subcutaneously into the hind legs of mice. NIR laser treatment was then applied to heat the area containing the gold nanorods to create increased localized heating and induce a heat-shock response for plasmid expression. This earlier study presented key limitations, e.g., the positive control failed to express in response to laser treatment, and the authors did not provide critical information in support of 
their claims in terms of sample size, variance, and statistical analysis. ${ }^{54}$ Conversely, the results from our present investigation demonstrate that the use of gold nanorods to impart localized heating does not represent a necessary requirement when using laser stimuli.

Following the encouraging results from the present study in HEK293T cells, which show a significant twofold increase in expression following laser stimulus, it is now very important to carry out more detailed studies to further improve this expression efficiency. While the present system was developed and tested in a mammalian cell line and has potential for use in a variety of disciplines, our goal is to continue developing this system for use in the reproductive sciences. In addition, investigations need to be explored in vivo to refine the use of laseractivated MSNP cargo in a model more suitable for studying reproductive development, such as the zebrafish or mouse embryo. Such studies are imperative since there are still challenges that need to be investigated comprehensively before practical applications in vivo or clinically. For example, most MSNP-based gene-delivery systems carry DNA or RNA on their outer surface, and studies have shown that this results in the exposure of cargo to enzyme-mediated degradation in vivo. ${ }^{55}$ Additionally, while MSNPs are known to biodegrade and excrete through the urinary system, the biological effect of undegraded MSNP products over a long period is still unknown. However, bioassays to determine such biodegradation properties are difficult due to the complexity of in situ detection of the degradation process in vivo, and the presence of natural silica species makes it difficult to distinguish the degraded products. ${ }^{51,55}$ Biosafety assays should extend from general toxicities against major organs and tissues to specific and specialized facets, such as neuro or reproductive toxicity. The impact of mesoporous silica species on mammalian embryos or embryonic development is unknown and is something our group is currently working to establish. This would eventually pave the way for translating this specialized nanocarrier system to human gametes and embryos.

\section{Conclusion}

The use of nanomaterials for investigative applications in reproductive biology has strong potential for expansion in the coming years. In the present study, a functional HSP promoter was fused to a GFP construct and successfully associated with nontoxic MSNPs, which were subsequently delivered into HEK293T cells. Furthermore, expression of the molecular cargo was increased in response to cellular heat-shock induced by IR laser irradiation. Our data therefore describe a method for increasing nanoparticle cargo expression in a spatial manner due to the heat-responsiveness of the cargo itself. These original investigations provide a useful foundation for future research into laser thermo-controlled MSNP nanocarriers with increased efficiency and control. This system could allow the delivery of cargo to inhibit, biomark, target, augment, or suppress pathways involved in the development of early embryos to study gene expression and development.

\section{Disclosures}

No conflicts of interest, financial or otherwise, are declared by the authors.

\section{Acknowledgments}

This research was funded by the Nuffield Department of Obstetrics and Gynaecology, University of Oxford, and by Clarendon Scholarships awarded to L.M.D, and N.B. Additional support was provided by Scatcherd European and Cyril and Phillis Long Scholarships awarded to N.B. The authors gratefully acknowledge Mike Julian and Jennifer Docherty from Research Instruments Life Sciences Ltd. for providing the Saturn 5 Active laser system.

\section{References}

1. N. Barkalina, C. Jones, and K. Coward, "Mesoporous silica nanoparticles: a potential targeted delivery vector for reproductive biology?" Nanomedicine 9, 557-560 (2014).

2. J. M. Rosenholm et al., "Nanoparticles in targeted cancer therapy: mesoporous silica nanoparticles entering preclinical development stage," Nanomedicine 7, 111-120 (2012).

3. I. I. Slowing et al., "Mesoporous silica nanoparticles as controlled release drug delivery and gene transfection carriers," $A d v$. Drug Delivery Rev. 60, 1278-1288 (2008).

4. R. A. Petros and J. M. DeSimone, "Strategies in the design of nanoparticles for therapeutic applications," Nat. Rev. Drug Discovery 9, 615627 (2010).

5. A. Kunzmann et al., "Toxicology of engineered nanomaterials: focus on biocompatibility, biodistribution and biodegradation," Biochim. Biophys. Acta 1810, 361-373 (2011).

6. D. Tarn et al., "Mesoporous silica nanoparticle nanocarriers: biofunctionality and biocompatibility," Acc. Chem. Res. 46, 792-801 (2013).

7. A. Albanese, P. S. Tang, and W. C. Chan, "The effect of nanoparticle size, shape, and surface chemistry on biological systems," Ann. Rev. Biomed. Eng. 14, 1-16 (2012).

8. J. G. Croissant et al., "Multifunctional gold-mesoporous silica nanocomposites for enhanced two-photon imaging and therapy of cancer cells," Front. Mol. Biosci. 3, 1 (2016).

9. X. Du et al., " $\gamma$-PGA-coated mesoporous silica nanoparticles with covalently attached prodrugs for enhanced cellular uptake and intracellular GSH-responsive release," Adv. Healthcare Mater. 4, 771-781 (2015).

10. W. Feng et al., "Effect of pH-responsive alginate/chitosan multilayers coating on delivery efficiency, cellular uptake and biodistribution of mesoporous silica nanoparticles based nanocarriers," ACS Appl. Mater. Interfaces 6, 8447-8460 (2014).

11. P. Shao et al., "The application of thermosensitive nanocarriers in controlled drug delivery," J. Nanomater. 2011, 17 (2011).

12. C. Yue et al., "Near-infrared light triggered ROS-activated theranostic platform based on Ce6-CPT-UCNPs for simultaneous fluorescence imaging and chemo-photodynamic combined therapy," Theranostics $\mathbf{6}$, 456-469 (2016).

13. V. Mamaeva et al., "Mesoporous silica nanoparticles as drug delivery systems for targeted inhibition of notch signaling in cancer," Mol. Ther. 19, 1538-1546 (2011).

14. L. Dai et al., "Redox-responsive nanocarrier based on heparin endcapped mesoporous silica nanoparticles for targeted tumor therapy in vitro and in vivo," Langmuir 30, 7867-7877 (2014).

15. S. M. Baek et al., "Triple hit with drug carriers: $\mathrm{pH}$ - and temperatureresponsive theranostics for multimodal chemo- and photothermaltherapy and diagnostic applications," ACS Appl. Mater. Interfaces 8(14), 8967-8979 (2016).

16. X. Li et al., "Mesoporous manganese silicate coated silica nanoparticles as multi-stimuli-responsive T1-MRI contrast agents and drug delivery carriers," Acta Biomater. 30, 378-387 (2016).

17. F. Martín-Saavedra and N. Vilaboa, "Remote patterning of transgene expression using near infrared-responsive plasmonic hydrogels," Optogenetics: Methods Protoc. 1408, 281-292 (2016).

18. Y. Zhang et al., "Polymeric prodrug grafted hollow mesoporous silica nanoparticles encapsulating near-infrared absorbing dye for potent combined photothermal-chemotherapy," ACS Appl. Mater. Interfaces 8, 6869-6879 (2016). 
19. R. I. Morimoto and M. G. Santoro, "Stress-inducible responses and heat shock proteins: new pharmacologic targets for cytoprotection," Nat. Biotechnol. 16, 833-838 (1998).

20. T. Deguchi et al., "Infrared laser-mediated local gene induction in medaka, zebrafish and Arabidopsis thaliana," Dev. Growth Differ. 51, 769-775 (2009).

21. E. Kimura et al., "Application of infrared laser to the zebrafish vascular system: gene induction, tracing, and ablation of single endothelial cells," Arterioscler. Thromb. Vasc. Biol. 33, 1264-1270 (2013).

22. N. Barkalina et al., "Effects of mesoporous silica nanoparticles upon the function of mammalian sperm in vitro," Nanomedicine 10, 859-870 (2014).

23. T. H. Chung et al., "The effect of surface charge on the uptake and biological function of mesoporous silica nanoparticles in 3T3-L1 cells and human mesenchymal stem cells," Biomaterials 28, 2959-2966 (2007).

24. D. Heinemann et al., "Gold nanoparticle mediated laser transfection for efficient siRNA mediated gene knock down," PLoS One 8, e58604 (2013).

25. D. Kim, Y. Y. Jeong, and S. Jon, "A drug-loaded aptamer-gold nanoparticle bioconjugate for combined CT imaging and therapy of prostate cancer," ACS Nano 4, 3689-3696 (2010).

26. V. F. Campos et al., "NanoSMGT: transgene transmission into bovine embryos using halloysite clay nanotubes or nanopolymer to improve transfection efficiency," Theriogenology 76, 1552-1560 (2011).

27. P. T. Yang et al., "In utero gene delivery using chitosan-DNA nanoparticles in mice," J. Surg. Res. 171, 691-699 (2011).

28. N. Barkalina et al., "Functionalization of mesoporous silica nanoparticles with a cell-penetrating peptide to target mammalian sperm in vitro," Nanomedicine 10, 1539-1553 (2015).

29. E. Aznar et al., "Finely tuned temperature-controlled cargo release using paraffin-capped mesoporous silica nanoparticles," Angew. Chem. Int. Ed. Engl. 50, 11172-11175 (2011).

30. X. Wu et al., "pH and thermo dual-stimuli-responsive drug carrier based on mesoporous silica nanoparticles encapsulated in a copolymer-lipid bilayer," ACS Appl. Mater. Interfaces 5, 10895-10903 (2013).

31. L. Chen et al., "Thermo-and $\mathrm{pH}$ dual-responsive mesoporous silica nanoparticles for controlled drug release," J. Controlled Release 213, e69-e70 (2015).

32. A. Schlossbauer et al., "A programmable DNA-based molecular valve for colloidal mesoporous silica," Angew. Chem. Int. Ed. Engl. 49, 47344737 (2010).

33. Z. Yu et al., "Temperature-responsive DNA-gated nanocarriers for intracellular controlled release," Chem. Соттип. 50, 3494-3497 (2014).

34. G. Martelli et al., "Coiled-coil peptide motifs as thermoresponsive valves for mesoporous silica nanoparticles," Chem. Сотmun. 49, 9932-9934 (2013).

35. J. Croissant and J. I. Zink, "Nanovalve-controlled cargo release activated by plasmonic heating," J. Am. Chem. Soc. 134, 7628-7631 (2012).

36. T. Xia et al., "Polyethyleneimine coating enhances the cellular uptake of mesoporous silica nanoparticles and allows safe delivery of siRNA and DNA constructs," ACS Nano 3, 3273-3286 (2009).

37. X. Li et al., "The packaging of siRNA within the mesoporous structure of silica nanoparticles," Biomaterials 32, 9546-9556 (2011).

38. J. Wang et al., "Increasing cellular uptake of mesoporous silica nanoparticles in human embryonic kidney cell line $293 \mathrm{~T}$ cells by using lipofectamine 2000," J. Biomed. Nanotechnol. 9, 1882-1890 (2013).

39. C. Grabher and J. Wittbrodt, "Efficient activation of gene expression using a heat-shock inducible Gal4/Vp16-UAS system in medaka," BMC Biotechnol. 4, 26 (2004).

40. W. Shoji and M. Sato-Maeda, "Application of heat shock promoter in transgenic zebrafish," Dev. Growth Differ. 50, 401-406 (2008).

41. A. Y. Sajjadi, K. Mitra, and M. Grace, "Expression of heat shock proteins 70 and 47 in tissues following short-pulse laser irradiation: assessment of thermal damage and healing," Med. Eng. Phys. 35, 1406-1414 (2013).

42. W. F. Zandberg et al., "Photothermal release of small molecules from gold nanoparticles in live cells," Nanomedicine 8, 908-915 (2012).

43. M. C. Halloran et al., "Laser-induced gene expression in specific cells of transgenic zebrafish," Development 127, 1953-1960 (2000).
44. D. M. Ramos et al., "Temporal and spatial control of transgene expression using laser induction of the HSP70 promoter," BMC Dev. Biol. 6, 55 (2006).

45. G. J. Wilmink et al., "In-vivo optical imaging of HSP70 expression to assess collateral tissue damage associated with infrared laser ablation of skin," J. Biomed. Opt. 13, 054066 (2008).

46. J. Zou et al., "Repression of heat shock transcription factor HSF1 activation by HSP90 (HSP90 complex) that forms a stress-sensitive complex with HSF1," Cell 94, 471-480 (1998).

47. D. V. Volodkin, A. G. Skirtach, and H. Möhwald, "Near-IR remote release from assemblies of liposomes and nanoparticles," Angew. Chem. Int. Ed. Engl. 48, 1807-1809 (2009).

48. S. Mura, J. Nicolas, and P. Couvreur, "Stimuli-responsive nanocarriers for drug delivery," Nat. Mater. 12, 991-1003 (2013).

49. N. T. Chen et al., "Enhanced plasmonic resonance energy transfer in mesoporous silica-encased gold nanorod for two-photon-activated photodynamic therapy," Theranostics 4, 798-807 (2014).

50. G. S. Terentyuk et al., "Laser-induced tissue hyperthermia mediated by gold nanoparticles: toward cancer phototherapy," J. Biomed. Opt. 14, 021016 (2009).

51. N. Barkalina et al., "Extracellular vesicle-mediated delivery of molecular compounds into gametes and embryos: learning from nature," Hum. Reprod. Update 21, 627-639 (2015).

52. A. M. Alkilany and C. J. Murphy, "Toxicity and cellular uptake of gold nanoparticles: what we have learned so far?" J. Nanopart. Res. 12, 2313-2333 (2010).

53. S. Abdelhamid et al., "Laser-induced modifications of gold nanoparticles and their cytotoxic effect," J. Biomed. Opt. 17, 068001 (2012).

54. H. A. Andersson et al., "HSP70 promoter-driven activation of gene expression for immunotherapy using gold nanorods and near infrared light," Vaccines 2, 216-227 (2014).

55. Y. Chen, H. Chen, and J. Shi, "In vivo bio-safety evaluations and diagnostic/therapeutic applications of chemically designed mesoporous silica nanoparticles," Adv. Mater. 25, 3144-3176 (2013).

Lien M. Davidson received her BSc (Hons) in biochemistry from Carleton University, Canada, and her MSc in clinical embryology from the University of Oxford. Currently, she is a PhD student at the Nuffield Department of Obstetrics and Gynaecology, University of Oxford, exploring the use of infrared lasers and mesoporous silica nanoparticle delivery platforms as investigative tools for developmental biology and early stage embryos.

Natalia Barkalina received her medical degree from Moscow Academy of Medicine. She received her MSc in clinical embryology and $\mathrm{PhD}$ in obstetrics and gynaecology from the University of Oxford. Her thesis work was based on the development of nanocarrier delivery platforms to engineer mammalian sperm.

Marc Yeste received his $\mathrm{PhD}$ in human reproduction from the Universitat de Girona, was a Marie Curie senior postdoctoral fellow in the Nuffield Department of Obstetrics and Gynaecology, University of Oxford, and is now a senior research fellow at the Universitat de Girona. His research focuses predominantly on the structure and function of mammalian sperm.

Celine Jones is the laboratory manager for the Coward Group and for the MSc in clinical embryology at the Nuffield Department of Obstetrics and Gynaecology, University of Oxford. She has a broad range of research interests, including the cellular, biochemical, and molecular mechanisms of oocyte activation.

Kevin Coward is the director of the Oxford MSc in clinical embryology and principal investigator at the Nuffield Department of Obstetrics and Gynaecology, University of Oxford. His research interests include oocyte activation deficiency, male factor infertility, the development of nanoparticle-mediated delivery systems for gametes and embryos, and the use of lasers in assisted reproductive technology, which incorporates collaboration with Research Instruments Ltd. 